\title{
A 27-Intersection Model for Representing Detailed Topological Relations between Spatial Objects in Two-Dimensional Space
}

\author{
Jingwei Shen ${ }^{1, *}$, Tinggang Zhou ${ }^{1}$ and Min Chen ${ }^{2}$ \\ 1 Chongqing Key Laboratory of Karst Environment, School of Geographical Sciences, Southwest University, \\ Chongqing 400715, China; zhoutg@swu.edu.cn \\ 2 Key Laboratory of Virtual Geographic Environment (Ministry of Education), Nanjing Normal University, \\ Nanjing 210046, Jiangsu, China; chenmin0902@163.com \\ * Correspondence: sjwgis@swu.edu.cn; Tel.: +86-23-6825-3912
}

Academic Editor: Wolfgang Kainz

Received: 9 December 2016; Accepted: 29 January 2017; Published: 5 February 2017

\begin{abstract}
Determining the spatial relations between objects is a primary function of a geographic information system (GIS). One important aspect of spatial relations is topological relations, which remain constant under topological transformations. Describing the geometry of a spatial object using the OpenGIS Simple Features Specification requires only simple features: the interior, boundary and exterior of a spatial object are defined. This paper proposes a comprehensive model, the 27-intersection model (27IM), which considers both the dimensions and the number of intersections. Some propositions are presented to exclude relations that the 27IM cannot implement. The 27IM describes six groups of topological relations: point/point, point/line, point/region, line/line, line/region and region/region. The formalism of the 27IM and the corresponding geometric interpretations between spatial objects are illustrated and then compared to the topological relations represented by the existing models, the nine-intersection model (9IM), the dimensionally-extended nine-intersection matrix (DE-9IM) and the separation number extended nine-intersection matrix (SNE-9IM). The results show that (1) the 27IM can represent the topological relations between two-dimensional spatial objects, (2) the 27IM can distinguish more topological relations than can the 9IM, DE-9IM or the SNE-9IM and that (3) the interoperability of the 27IM with the 9IM, DE-9IM and SNE-9IM is good.
\end{abstract}

Keywords: topological relation; 27IM; dimension; separation

\section{Introduction}

The spatial relations between spatial entities are as important as the entities themselves [1]. A spatial relation specifies how an object is located in space in relation to some reference object. Topological relations, distance relations and orientation relations are commonly used types of spatial relations. Topological properties are the most fundamental in spatial language and spatial cognition when compared to Euclidean, metric and vector spaces [2-4]. Topological relations remain constant when the coordinate space is deformed, such as by twisting or stretching. Consequently, the topological relations of spatial objects are commonly used in GIS. Simple queries, such as "Which universities are located in this city?" or "Are these two land parcels disjoint?" are widely addressed, while more complex queries, such as "How many times does the river cross this state's boundary?" and "What are the dimension of these intersections?" require further analysis.

Previous studies have investigated the formalization of topological relations, such as intervalbased temporal logic [5,6], point-set topology [1,7-9] and region connection calculus (RCC) [10-15]. 
In the scope of this paper, point-set topology, which is the study of the general abstract nature of continuity or the connectedness of spaces, has been used to discuss the formalization of topological relations.

Over the past three decades, research to formalize topological relations based on point-set topology has been conducted, and the four-intersection model (4IM) [7], the nine-intersection model (9IM) [8], the Voronoi-based nine-intersection model [1], the intersection and difference model [16], an extended model expressed as $4 \times 4$ matrices [17], the $9^{+}$-intersection model [18], the uncertain intersection and difference model [19] and the double straight line four-intersection models [20] have been proposed. All of the above models consider the content invariant (i.e., the emptiness or non-emptiness) of the intersections. The 4IM and 9IM were compared on spatial relations, and the result was that 9IM with the content invariant provides more details than does the 4IM [9]. A complete classification of spatial relations was demonstrated using the Voronoi-based nine-intersection model [21]. Topological relations for spatial scene [22,23] and compound spatial objects [24] were also presented. We can conclude that the 4IM and 9IM, as well as extended models based on these have been widely studied.

In addition to the content invariant, other topological invariants have been used to further describe topological relations details. Many refinements of the empty/non-empty intersections have been proposed. For instance, the dimension of intersections [25], the number of separations in intersections [26], four invariants, including the dimension of the components, their types, their relationships with respect to the exterior neighborhood and the sequence of the components [27], three invariants, including intersection sequence, intersection type and collinearity sense for lines [28] and a set of hierarchically topological invariants, including content, separation number, dimension, element type and sequence [16], has been proposed. These methods are refinements of the existing 4IM and 9IM and can represent more topological relation details whenever necessary.

In recent years, topological relationships from metric refinements have been discussed in the GIS literature, such as splitting ratios and closeness measures [29], splitting, closeness and approximate alongness for line-region relations [30] and three content measures to characterize spatial configurations [31]. Moreover, the 9IM has been extended by capturing the metric details for line-line relations through splitting ratios and closeness measures [32], and a comprehensive set of eleven metric refinements [33], a distance based interpretation for mereotopology [34] and expansion closeness and contraction closeness [35] have been investigated over the past few years. Metric refinements are the refinement of the intersection model and can provide additional information for topological relations. Metric refinements represent ranges of values that together claim a topological relation.

The scope of this paper includes the definition of a formalism (model) to describe the topological relations of spatial objects embedded in the plane. To describe the details of topological relations, a comprehensive model for topological spatial relations is proposed, and two topological invariants are considered: (1) the dimension of the intersections and (2) the number of separations.

The remainder of this paper presents the proposed comprehensive model for topological spatial relations used to specify the dimension and the number of separations. Section 2 introduces the model. Section 3 describes six groups of topological relations described by the 27IM. Section 4 discusses the refinement and interoperability of the 27IM, and Section 5 provides conclusions and discusses future work.

\section{Methods}

\subsection{Spatial Data Model}

To describe the geometry of spatial objects, many spatial data models, such as the simplicial complex [36], the three-dimensional formal data structure [37], the tetrahedron network [38,39], the cell complex [40,41], the geometry object model [42], the simplified spatial model [43], the multi-level model [44], the combinatorial data model [45], the common information model [46] and the geometric algebra model $[47,48]$, have been proposed. Generally, these models maintain four geometric 
abstractions of a real object: point, line, surface and body. The differences between these models lie in their spatial object's components and their mode of organization. The geometric object model defined by the OpenGIS Simple Features Specification has been widely accepted for two-dimensional spatial objects. In this paper, the definition of spatial objects in two-dimensional space refers to the OpenGIS Simple Features Specification.

Point set theory provides a framework for modeling the intersection of two spatial objects. Every spatial object is characterized by the following spatial attributes: an interior, a boundary and an exterior. The definitions of interior, boundary and exterior are given below [49].

Definition 1. Let A be a subset of a topological space X. The interior of $\mathrm{A}$ is the union of all open sets contained in $\mathrm{A}$. The closure of $\mathrm{A}$ is the intersection of all closed sets containing $\mathrm{A}$.

Definition 2. Let A be a subset of a topological space X. The boundary of A is the set of the closure of A minus the interior of $\mathrm{A}$.

Definition 3. Let $\mathrm{A}$ be a subset of a topological space $\mathrm{X}$. The exterior of $\mathrm{A}$ is the set of $\mathrm{X}$ minus the closure of $\mathrm{A}$.

Based on the OpenGIS Simple Features Specification and algebraic topology, definitions and their interior, boundary and exterior of point, line and region are given as follows (Figure 1).

A point is a 0-dimensional geometry and represents a single location in coordinate space (Figure 1a). The interior of a point is a point; the boundary of a point is an empty set; and the exterior of a point includes everything that is not the point.

A line is a one-dimensional geometric object usually stored as a sequence of points with linear interpolation between the points (Figure 1b). A line never passes through the same point twice. The interior of a line is a line without its endpoints; the boundary of a line is its two endpoints; and the exterior of a line is a set of points that does not contain the line.

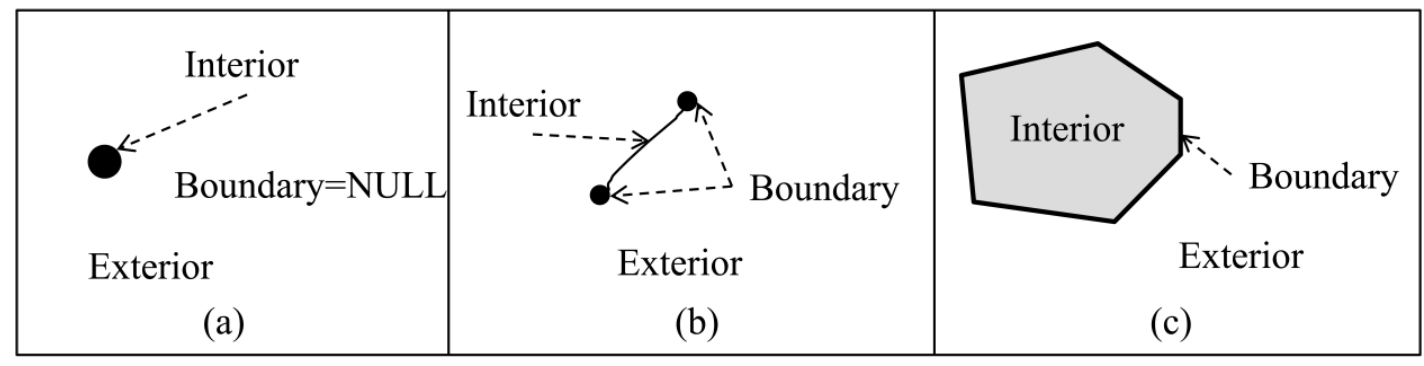

Figure 1. Spatial objects including interior, boundary and exterior. (a) A point with its interior, boundary and exterior. (b) A line with its interior, boundary and exterior. (c) A region with its interior, boundary and exterior.

A region is a closed 2-dimensional geometric object defined by one exterior boundary (Figure 1c). The interior of the region is the set of points inside the boundary; the boundary of a region is the lines forming the boundary, and the exterior of a region is a set of points that do not include the region.

According to the dimensions of spatial objects, there are six groups of topological relations: point/point, point/line, point/region, line/line, line/region and region/region relations, which will be described.

\subsection{Different Topological Invariants for Topological Relations}

Topological relations between two spatial objects, $A$ and $B$, are based on the intersection of $A^{\prime} \mathrm{s}$ interior, boundary and exterior with $B^{\prime}$ s interior, boundary and exterior. The nine intersections between 
$A$ and $B$ can be described by a topological relation using a $3 \times 3$ matrix. The content, the dimension and the number of separation invariants are used to describe topological relations. Taking the spatial objects in Figure 2 as examples, their topological relations can be described by following topological relation models.

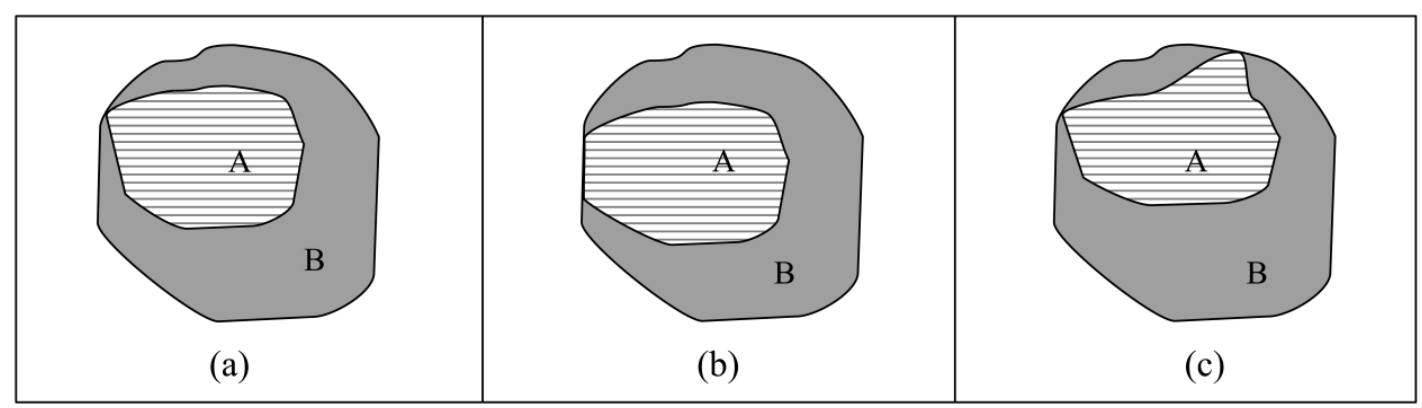

Figure 2. $(\mathbf{a}-\mathbf{c})$ Spatial objects and their topological relations.

A $3 \times 3$ matrix with empty and non-empty values is represented by Equation (1). $A^{\prime}$ s interior, $A^{\prime}$ 's boundary, $A^{\prime}$ s exterior, $B^{\prime}$ s interior, $B^{\prime}$ s boundary and $B^{\prime}$ s exterior are denoted by $A^{0}, \partial A, A^{-}$, $B^{o}, \partial B$ and $B^{-}$, respectively. By considering the empty and non-empty values, $512\left(512=2^{9}\right)$ binary topological relations can be distinguished in theory. However, not all 512 binary topological relations can be implemented. The 9IM can distinguish 33 relations between two simple lines, 19 relations between a simple line and a region and 8 relations between two simple regions [8]. The value of the intersection in Equation (1) can be drawn from $\{0,1\}$, where a 0 means that the intersection is null and a 1 represents that the intersection is not null,

$$
R_{9 I M}(A, B)=\left[\begin{array}{ccc}
A^{o} \cap B^{o} & A^{o} \cap \partial B & A^{o} \cap \mathrm{B}^{-} \\
\partial A \cap B^{o} & \partial A \cap \partial B & \partial A \cap \mathrm{B}^{-} \\
\mathrm{A}^{-} \cap B^{o} & \mathrm{~A}^{-} \cap \partial B & \mathrm{~A}^{-} \cap \mathrm{B}^{-}
\end{array}\right]
$$

Based on the 9IM, the topological relations between A and B are described below. The formalisms of the topological relations in Figure 2a-c expressed by the 9IM are all the same. In other words, all of the topological relations in Figure 2a-c can be described as follows:

$$
R_{9 I M}(A, B)=\left[\begin{array}{lll}
1 & 0 & 0 \\
1 & 1 & 0 \\
1 & 1 & 1
\end{array}\right]
$$

A dimensionally-extended nine-intersection matrix (DE-9IM) is represented by Equation (3). The dimension invariant is used to distinguish the dimension of the intersection and is not higher than the lowest dimension of the two spatial objects. Therefore, the largest value of the dimension of the intersection is 2 between simple points, lines and regions. The dimension of the intersection is -1 if the intersection is empty. The dimension of a non-empty intersection is taken to be the maximum of the dimensions of the intersection. For example, if the intersections contain both points and lines, the dimension of the intersection is set to 1 . Considering the dimensions of intersections, 262,144 $\left(262,144=4^{9}\right)$ topological relations can be distinguished in theory. However, not all 262,144 topological relations can be implemented, and some topological relations have no practical significance.

$$
R_{D E-9 I M}(A, B)=\left[\begin{array}{lll}
\operatorname{dim}\left(A^{o} \cap B^{o}\right) & \operatorname{dim}\left(A^{o} \cap \partial B\right) & \operatorname{dim}\left(A^{o} \cap B^{-}\right) \\
\operatorname{dim}\left(\partial A \cap B^{o}\right) & \operatorname{dim}(\partial A \cap \partial B) & \operatorname{dim}\left(\partial A \cap B^{-}\right) \\
\operatorname{dim}\left(A^{-} \cap B^{o}\right) & \operatorname{dim}\left(A^{-} \cap \partial B\right) & \operatorname{dim}\left(A^{-} \cap B^{-}\right)
\end{array}\right]
$$


Based on the DE-9IM, the topological relations between $A$ and $B$ are described below. The formalisms of topological relations in Figure 2a,c expressed by the DE-9IM are the same (Equation (4)), whereas the formalism of topological relations in Figure 2b (Equation (5)) is different from that of Figure 2a,c. Compared with the 9IM, the DE-9IM can not only distinguish whether two sets intersect, but also the dimensions of the intersections:

$$
\begin{aligned}
& R_{D E-9 I M}(A, B)=\left[\begin{array}{ccc}
2 & -1 & -1 \\
1 & 0 & -1 \\
2 & 1 & 2
\end{array}\right] \\
& R_{D E-9 I M}(A, B)=\left[\begin{array}{ccc}
2 & -1 & -1 \\
1 & 1 & -1 \\
2 & 1 & 2
\end{array}\right]
\end{aligned}
$$

Short for a $3 \times 3$ matrix that shows the number of separations, the separation number extended nine-intersection matrix (SNE-9IM) is represented by Equation (6). The number of separations is used to express the number of connected components. The measure for the separation is the topological invariant of the Euler-Poincare characteristic [26], but the value may be negative. The number of separations is defined as a positive number in this study. Because the number of separations can be any integer greater or equal to 0 , in theory, the SNE-9IM can distinguish innumerable topological relations:

$$
R_{S N E-9 I M}=\left[\begin{array}{lll}
\chi\left(A^{o} \cap B^{o}\right) & \chi\left(A^{o} \cap \partial B\right) & \chi\left(A^{o} \cap B^{-}\right) \\
\chi\left(\partial A \cap B^{o}\right) & \chi(\partial A \cap \partial B) & \chi\left(\partial A \cap B^{-}\right) \\
\chi\left(A^{-} \cap B^{o}\right) & \chi\left(A^{-} \cap \partial B\right) & \chi\left(A^{-} \cap B^{-}\right)
\end{array}\right]
$$

Based on the SNE-9IM, the topological relations between $A$ and $B$ are described below. The formalisms of the topological relations in Figure 2a,b expressed by the SNE-9IM are identical (Equation (7)), whereas the formalism of the topological relations in Figure 2c (Equation (8)) is different from that of Figure 2a,b. Compared with the 9IM, the SNE-9IM can not only distinguish whether two sets intersect, but also the number of separations.

$$
\begin{aligned}
& R_{S N E-9 I M}(A, B)=\left[\begin{array}{lll}
1 & 0 & 0 \\
1 & 1 & 0 \\
1 & 1 & 1
\end{array}\right] \\
& R_{S N E-9 I M}(A, B)=\left[\begin{array}{lll}
1 & 0 & 0 \\
1 & 2 & 0 \\
1 & 1 & 1
\end{array}\right]
\end{aligned}
$$

\subsection{A Comprehensive Model for Topological Relations}

Both the dimensions and the number of separations are taken into account in the proposed comprehensive model. This comprehensive model represents the topological spatial relations between two spatial objects, $A$ and $B$, based on a consideration of the number of separations of the interiors, boundaries and exteriors of $A$ and $B$ in each dimension. The framework for this comprehensive model is the ordered set of the 27 intersections, called the 27-intersection model (27IM), which is concisely represented as a $9 \times 3$ matrix (Equation (9)).

$$
R_{27 I M}(A, B)=\left[\begin{array}{ccc}
\chi_{0,1,2}\left(A^{o} \cap B^{o}\right) & \chi_{0,1,2}\left(A^{o} \cap \partial B\right) & \chi_{0,1,2}\left(A^{o} \cap B^{-}\right) \\
\chi_{0,1,2}\left(\partial A \cap B^{o}\right) & \chi_{0,1,2}(\partial A \cap \partial B) & \chi_{0,1,2}\left(\partial A \cap B^{-}\right) \\
\chi_{0,1,2}\left(A^{-} \cap B^{o}\right) & \chi_{0,1,2}\left(A^{-} \cap \partial B\right) & \chi_{0,1,2}\left(A^{-} \cap B^{-}\right)
\end{array}\right]
$$


Every member in Equation (9) contains three intersections, for example, $\chi_{0,1,2}\left(A^{o} \cap B^{o}\right)$ represents the intersection of $\chi_{0}\left(A^{o} \cap B^{o}\right), \chi_{1}\left(A^{o} \cap B^{o}\right)$ and $\chi_{2}\left(A^{o} \cap B^{o}\right)$. Here, $\chi_{0}\left(A^{o} \cap B^{o}\right), \chi_{1}\left(A^{o} \cap B^{o}\right)$ and $\chi_{2}\left(A^{o} \cap B^{o}\right)$ separately represent the number of the separations in 0 dimensions, 1 dimension and 2 dimensions. Equation (9) can also be represented as a twenty-seven-tuple, i.e., $\chi_{0}\left(A^{o} \cap B^{o}\right)$, $\chi_{1}\left(A^{o} \cap B^{o}\right), \chi_{2}\left(A^{o} \cap B^{o}\right), \chi_{0}\left(A^{o} \cap \partial B\right), \chi_{1}\left(A^{o} \cap \partial B\right), \chi_{2}\left(A^{o} \cap \partial B\right), \chi_{0}\left(A^{o} \cap B^{-}\right), \chi_{1}\left(A^{o} \cap B^{-}\right)$, $\chi_{2}\left(A^{o} \cap B^{-}\right), \chi_{0}\left(\partial A \cap B^{o}\right), \chi_{1}\left(\partial A \cap B^{o}\right), \chi_{2}\left(\partial A \cap B^{o}\right), \chi_{0}(\partial A \cap \partial B), \chi_{1}(\partial A \cap \partial B), \chi_{2}(\partial A \cap \partial B)$, $\chi_{0}\left(\partial A \cap B^{-}\right), \chi_{1}\left(\partial A \cap B^{-}\right), \chi_{2}\left(\partial A \cap B^{-}\right), \chi_{0}\left(A^{-} \cap B^{o}\right), \chi_{1}\left(A^{-} \cap B^{o}\right), \chi_{2}\left(A^{-} \cap B^{o}\right), \chi_{0}\left(A^{-} \cap \partial B\right)$, $\chi_{1}\left(A^{-} \cap \partial B\right), \chi_{2}\left(A^{-} \cap \partial B\right), \chi_{0}\left(A^{-} \cap B^{-}\right), \chi_{1}\left(A^{-} \cap B^{-}\right)$and $\chi_{2}\left(A^{-} \cap B^{-}\right)$. The value of the above intersections records the number of separations in each dimension.

The 27IM is a general framework for representing the relations between two spatial objects in two-dimensional space. However, it cannot represent all of the possible intersections; therefore, some propositions are proposed to exclude the relations that cannot be represented.

Proposition 1. The dimension of two sets is no greater than the smallest dimension of the sets (Equation (10)). In Equation (10), $S_{1}$ and $S_{2}$ are two sets, and $\operatorname{dim}\left(S_{1}\right), \operatorname{dim}\left(S_{2}\right)$ and $\operatorname{dim}\left(S_{1} \cap S_{2}\right)$ are separately the dimension of the $S_{1}, S_{2}$ and the intersection of $S_{1}$ and $S_{2}$.

$$
\operatorname{dim}\left(S_{1} \cap S_{2}\right) \leq \min \left(\operatorname{dim}\left(S_{1}\right), \operatorname{dim}\left(S_{2}\right)\right)
$$

Proof. If one set is $S_{1}$ and the other set is $S_{2}$, the intersection is not only a subset of $S_{1}$, but also a subset of $S_{2}$. Therefore, the dimension of the intersection is no greater than the dimension of $S_{1}$ or $S_{2}$.

Proposition 2. The value of the dimension of the intersection is constrained by the types of two spatial objects.

Condition 1. If the intersection is not null, the only possible value for a line's interior and a region's interior is drawn from \{1\}. In Equation (11), $L^{o}$ and $R^{o}$ are separately the interior of the line and the interior of the region, and $\operatorname{dim}\left(L^{o} \cap R^{o}\right)$ is the dimension of the intersection of $L^{o}$ and $R^{o}$.

$$
\operatorname{dim}\left(L^{o} \cap R^{o}\right)=1\left(L^{o} \cap R^{o} \neq \phi\right)
$$

Proof. If the interior of a line intersects with a region's interior, the dimension of the intersection can only be drawn from $\{0,1\}$ according to Proposition 1 . If a point $x$ belongs to both the interior of a line and a region, the neighborhood of $x$ must be in the interior of the region. Moreover, a subset of the line's interior must be in the neighborhood of $x$. The dimension of the subset of line's interior is 1.

Condition 2. If the intersection is not null, the only possible value for one region's interior and other region's interior is drawn from \{2\}. In Equation (12), $R_{1}^{o}$ and $R_{2}^{o}$ are the interior of two regions, and $\operatorname{dim}\left(R_{1}^{o} \cap R_{2}^{o}\right)$ is the dimension of the intersection of $R_{1}^{o}$ and $R_{2}^{o}$.

$$
\operatorname{dim}\left(R_{1}^{o} \cap R_{2}^{o}\right)=2\left(R_{1}^{o} \cap R_{2}^{o} \neq \phi\right)
$$

Proof. If the interior of a region intersects with another region's interior, the dimension of the intersection is drawn from $\{0,1,2\}$ according to Proposition 1 . If a point $x$ belongs to the interior of two regions, the neighborhood of $x$ must be in the interior of both regions. Therefore, the intersection of two regions can only be a region when the intersection is not null.

Proposition 3. The dimension of the intersection between two exteriors is drawn from $\{2\}$.

Proof. $A^{-}$or $B^{-}$is the set of all points in the embedding space $R^{2}$ not contained in $A$ or $B$. Because $A$, $B$ and $A \cup B$ are subsets of $R^{2}, A^{-}$or $B^{-}$are two-dimensional, and $A^{-}$and $B^{-}$must have intersections. The dimension of the intersection is drawn from $\{0,1,2\}$ according to Proposition 1 . If a point $x$ belongs to the exterior of $A$ and $B$, the neighborhood of $x$ must be in the exterior of $A$ or $B$; therefore, the dimension of two regions' intersection can only be two-dimensional. 
Proposition 4. The dimension of the intersection between the boundary of a point and other sets is null.

Proof. Because the boundary of a point is an empty set, the intersection between the boundary of a point and another set must be an empty set; therefore, the dimension of the intersection is null.

Based on the above propositions, Table 1 lists the possible tuples for the six groups of topological relations: point/point, point/line, point/region, line/line, line/region and region/region. If the tuples can be implemented, the corresponding cell in Table 1 contains a checkmark (" $\sqrt{ }$ "); otherwise, the cell contains a " $\times$ ".

Table 1. Possible tuples for six groups of topological relations.

\begin{tabular}{|c|c|c|c|c|c|c|}
\hline & Point/Point & Point/Line & Point/Region & Line/Line & Line/Region & Region/Region \\
\hline$\chi_{0}\left(A^{o} \cap B^{o}\right)$ & $\sqrt{ }$ & $\sqrt{ }$ & $\sqrt{ }$ & $\sqrt{ }$ & $\times$ & $x$ \\
\hline$\chi_{1}\left(A^{o} \cap B^{o}\right)$ & $\times$ & $\times$ & $\times$ & $\sqrt{ }$ & $\sqrt{ }$ & $x$ \\
\hline$\chi_{2}\left(A^{o} \cap B^{o}\right)$ & $\times$ & $\times$ & $\times$ & $\times$ & $\times$ & $\sqrt{ }$ \\
\hline$\chi_{0}\left(A^{o} \cap \partial B\right)$ & $\times$ & $\sqrt{ }$ & $\sqrt{ }$ & $\sqrt{ }$ & $\sqrt{ }$ & $\times$ \\
\hline$\chi_{1}\left(A^{o} \cap \partial B\right)$ & $x$ & $\times$ & $\times$ & $\times$ & $\sqrt{ }$ & $\sqrt{ }$ \\
\hline$\chi_{2}\left(A^{o} \cap \partial B\right)$ & $x$ & $\times$ & $\times$ & $\times$ & $x$ & $\times$ \\
\hline$\chi_{0}\left(A^{o} \cap B^{-}\right)$ & $\sqrt{ }$ & $\sqrt{ }$ & $\sqrt{ }$ & $\times$ & $\times$ & $\times$ \\
\hline$\chi_{1}\left(A^{o} \cap B^{-}\right)$ & $\times$ & $\times$ & $\times$ & $\sqrt{ }$ & $\sqrt{ }$ & $x$ \\
\hline$\chi_{2}\left(A^{o} \cap B^{-}\right)$ & $x$ & $x$ & $\times$ & $\times$ & $x$ & $\sqrt{ }$ \\
\hline$\chi_{0}\left(\partial A \cap B^{o}\right)$ & $x$ & $x$ & $\times$ & $\sqrt{ }$ & $\sqrt{ }$ & $\times$ \\
\hline$\chi_{1}\left(\partial A \cap B^{o}\right)$ & $x$ & $\times$ & $x$ & $\times$ & $x$ & $\sqrt{ }$ \\
\hline$\chi_{2}\left(\partial A \cap B^{o}\right)$ & $x$ & $x$ & $x$ & $\times$ & $\times$ & $\times$ \\
\hline$\chi_{0}(\partial A \cap \partial B)$ & $x$ & $\times$ & $\times$ & $\sqrt{ }$ & $\sqrt{ }$ & $\sqrt{ }$ \\
\hline$\chi_{1}(\partial A \cap \partial B)$ & $x$ & $x$ & $x$ & $\times$ & $\times$ & $\sqrt{ }$ \\
\hline$\chi_{2}(\partial A \cap \partial B)$ & $x$ & $x$ & $x$ & $x$ & $x$ & $\times$ \\
\hline$\chi_{0}\left(\partial A \cap B^{-}\right)$ & $\times$ & $x$ & $x$ & $\sqrt{ }$ & $\sqrt{ }$ & $\times$ \\
\hline$\chi_{1}\left(\partial A \cap B^{-}\right)$ & $x$ & $x$ & $x$ & $\times$ & $x$ & $\sqrt{ }$ \\
\hline$\chi_{2}\left(\partial A \cap B^{-}\right)$ & $x$ & $x$ & $\times$ & $x$ & $\times$ & $x$ \\
\hline$\chi_{0}\left(A^{-} \cap B^{o}\right)$ & $\sqrt{ }$ & $x$ & $x$ & $x$ & $\times$ & $x$ \\
\hline$\chi_{1}\left(A^{-} \cap B^{o}\right)$ & $\times$ & $\sqrt{ }$ & $x$ & $\sqrt{ }$ & $\times$ & $\times$ \\
\hline$\chi_{2}\left(A^{-} \cap B^{o}\right)$ & $\times$ & $\times$ & $\sqrt{ }$ & $\times$ & $\sqrt{ }$ & $\sqrt{ }$ \\
\hline$\chi_{0}\left(A^{-} \cap \partial B\right)$ & $x$ & $\sqrt{ }$ & $x$ & $\sqrt{ }$ & $x$ & $\times$ \\
\hline$\chi_{1}\left(A^{-} \cap \partial B\right)$ & $\times$ & $\times$ & $\sqrt{ }$ & $x$ & $\sqrt{ }$ & $\sqrt{ }$ \\
\hline$\chi_{2}\left(A^{-} \cap \partial B\right)$ & $\times$ & $\times$ & $\times$ & $\times$ & $\times$ & $\times$ \\
\hline$\chi_{0}\left(A^{-} \cap B^{-}\right)$ & $x$ & $\times$ & $\times$ & $\times$ & $x$ & $x$ \\
\hline$\chi_{1}\left(A^{-} \cap B^{-}\right)$ & $\times$ & $\times$ & $\times$ & $\times$ & $\times$ & $\times$ \\
\hline$\chi_{2}\left(A^{-} \cap B^{-}\right)$ & $\sqrt{ }$ & $\sqrt{ }$ & $\sqrt{ }$ & $\sqrt{ }$ & $\sqrt{ }$ & $\sqrt{ }$ \\
\hline
\end{tabular}

\section{Results}

Three types of spatial objects, including point, line and region, are defined; therefore, six groups of topological relations are summarized. These six groups of topological relations are point/point, point/line, point/region, line/line, line/region and region/region relations, and they are described by the 27IM in this study.

First, point/point topological relations are described. Because the boundary of a point is empty, one point may be disjoint or equal to another point. Therefore, two topological relations can be realized between two points. Figure 3 shows the formalism of the 27IM and the corresponding geometric interpretations of the two relations between two points. 


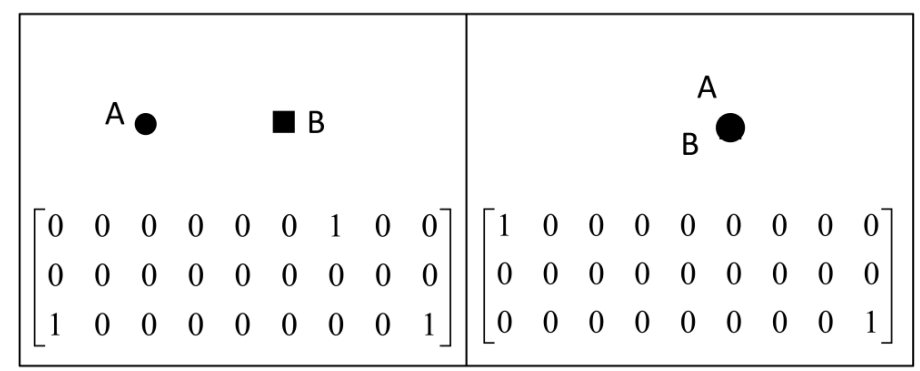

Figure 3. The formalism of the 27-intersection model (27IM) and the corresponding geometric interpretations between two points.

Second, point/line topological relations are described. Because the dimension of a point is lower than that of a line, the relation between a point and a line may be disjoint, meet or within. Therefore, there are three topological relations between a point and a line. Figure 4 shows the formalism of the 27IM and the corresponding geometric interpretations of the three relations between a point and a line.

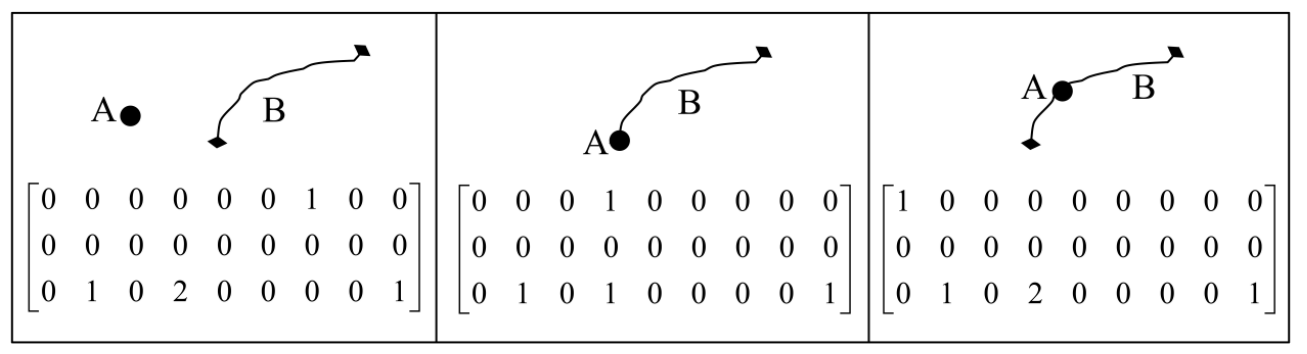

Figure 4. The formalism of the 27IM and the corresponding geometric interpretations between a point and a line.

Third, point/region topological relations are described. Similar to point/line topological relations, there are three topological relations between a point and a region. Figure 5 shows the formalism of the 27IM and the corresponding geometric interpretations of the three relations between a point and a region.

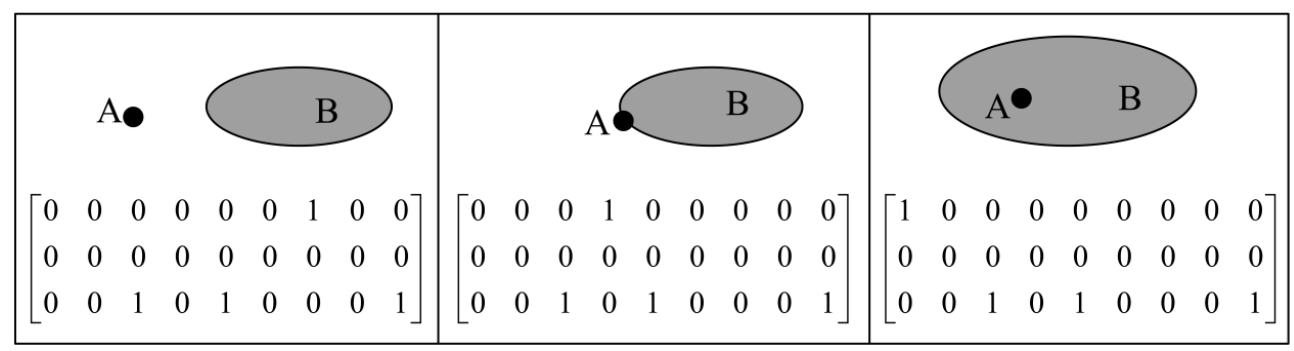

Figure 5. The formalism of the 27IM and the corresponding geometric interpretations between a point and a region.

Fourth, line/line topological relations are described. Considering the dimension and the number of separations (for example, the number of intersections between the interior of two lines may be infinite), there are countless theoretical topological relations between two lines; therefore, it is impossible to express all of the possible topological relations. Instead, Figure 6 shows the formalism of the 27IM and the corresponding geometric interpretations of nine typical relations between two lines. 


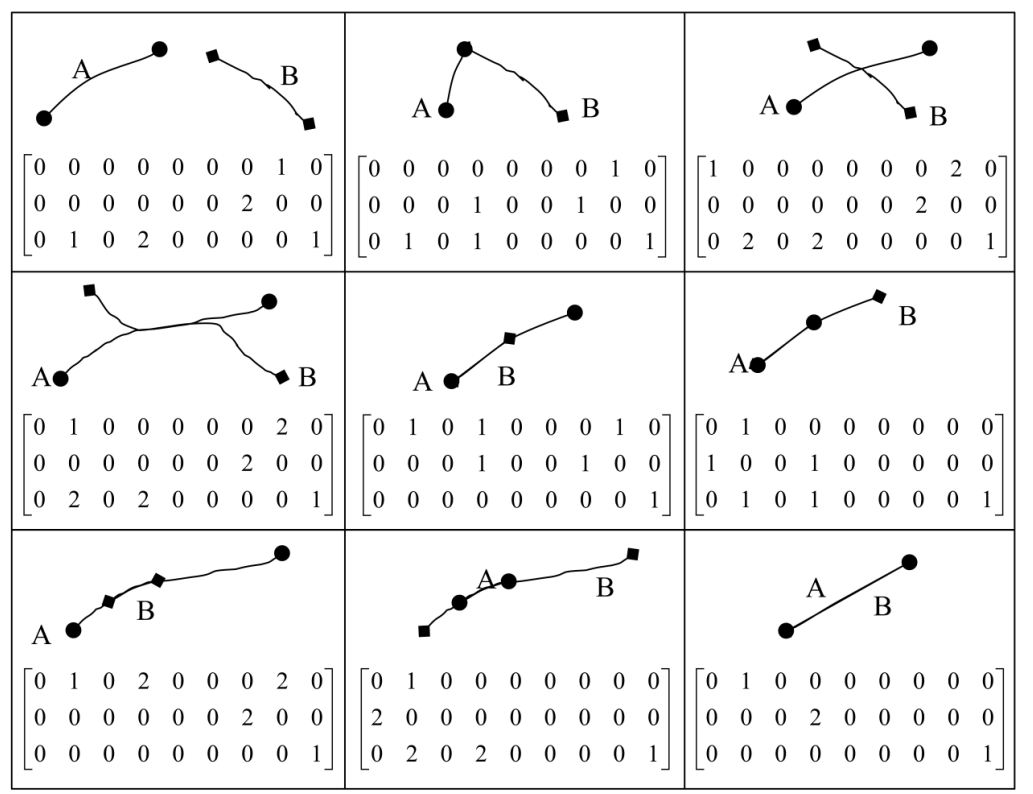

Figure 6. The formalism of the 27IM and corresponding geometric interpretations between two lines.

Fifth, line/region topological relations are described. Considering dimensions and the number of separations (for example, the number of intersections between the interior of a line and the boundary of a region, the number of intersections between the interior of a line and the interior of a region and the number of intersections between the interior of a line and the exterior of a region may be infinite), there are countless topological relations between a line and a region; therefore, it is impossible to express all of the possible topological relations. Instead, Figure 7 shows the formalism of the 27IM and the corresponding geometric interpretations of nine typical relations between a line and a region.

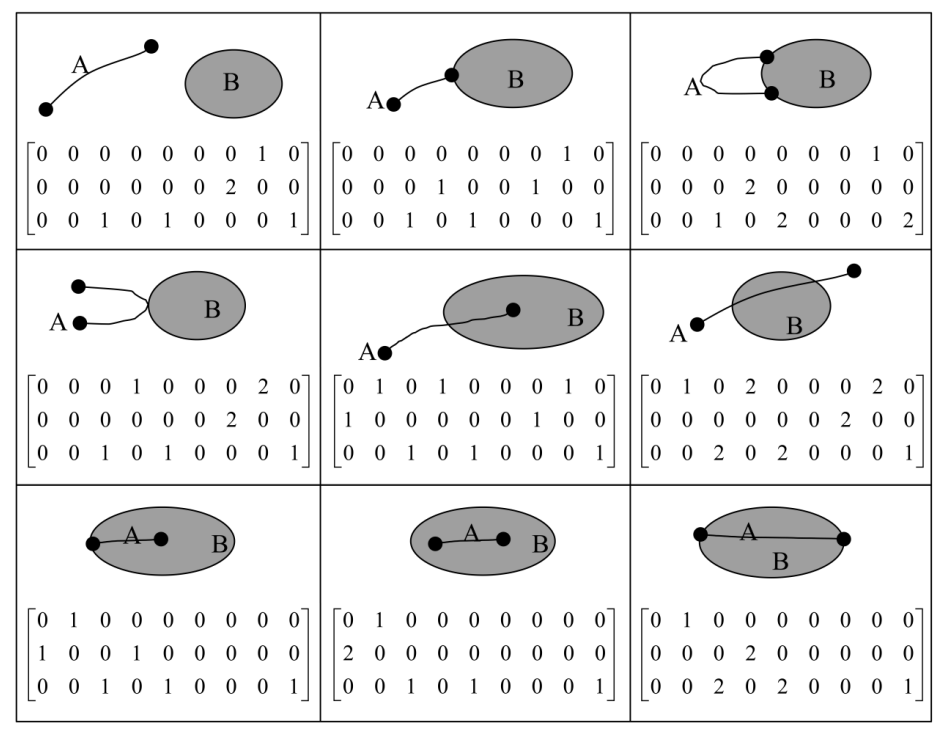

Figure 7. The formalism of the 27IM and the corresponding geometric interpretations between a line and a region.

Sixth, region/region topological relations are described. Considering dimensions and the number of separations (for instance, the number of intersections between the interior of two regions and the number of intersections between the boundaries of two regions may be infinite), there are countless topological relations between two regions; therefore, it is impossible to express all of the possible 
topological relations. Instead, Figure 8 shows the formalism of the 27IM and the corresponding geometric interpretations of nine typical relations between two regions.

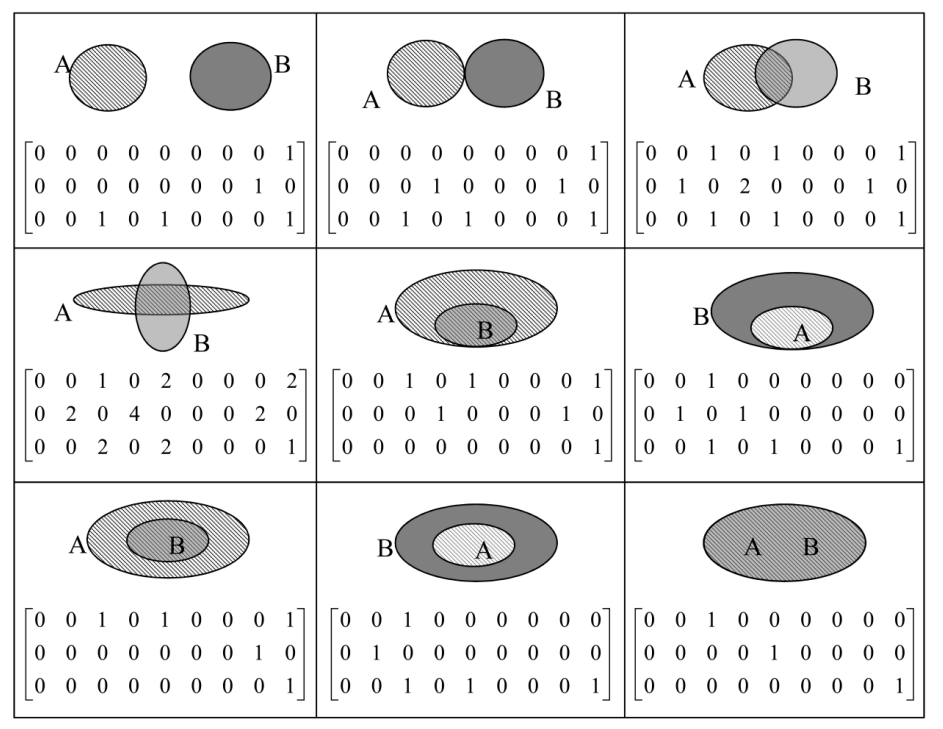

Figure 8. The formalism of the 27IM and the corresponding geometric interpretations between two regions.

\section{Discussion}

\subsection{The Refinement of Topological Relations}

Many topological relation models have been proposed; therefore, it is useful to compare the proposed model and other models. By considering the dimension and the number of separations, detailed topological relations are distinguished. Some instances, including line/line, line/region and region/region, are given in Figure 9.

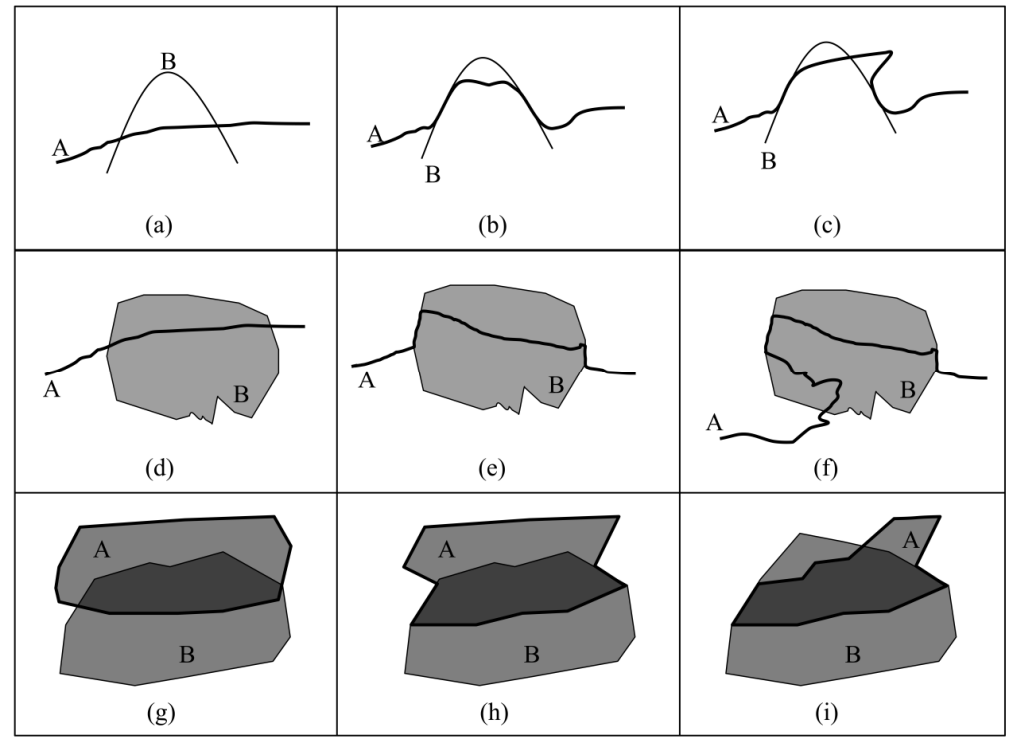

Figure 9. Examples of the topological relations between two spatial objects. (a-c) Topological relations between Line A and Line B. (d-f) Topological relations between line A and region B. (g-i) topological relations between Region A and Region B. 
Three widely-used topological relation representation models, the 9IM, DE-9IM and SNE-9IM, as well as the proposed 27IM are compared regarding their descriptions of the details of topological relations in this study. Table 2 lists the results of comparing the relations in Figure 9 using the 9IM, DE-9IM, SNE-9IM and 27IM.

Table 2. Topological relation as described by the 9IM, dimensionally-extended nine-intersection matrix (DE-9IM), separation number extended nine-intersection matrix (SNE-9IM) and 27IM.

\begin{tabular}{|c|c|c|c|c|c|c|c|c|c|c|c|c|c|c|c|c|c|c|}
\hline & \multicolumn{3}{|c|}{ 9IM } & \multicolumn{3}{|c|}{ DE-9IM } & \multicolumn{3}{|c|}{ SNE-9IM } & \multicolumn{9}{|c|}{ 27IM } \\
\hline \multirow{4}{*}{ Figure 9a } & 1 & 0 & 1 & 0 & & 1 & 2 & 0 & 3 & 2 & 0 & 0 & 0 & 0 & 0 & 0 & 3 & 0 \\
\hline & 0 & 0 & 1 & -1 & -1 & 0 & 0 & 0 & 2 & 0 & 0 & 0 & 0 & 0 & 0 & 2 & 0 & 0 \\
\hline & 1 & 1 & 1 & 1 & 0 & 2 & 3 & 2 & 2 & 0 & 3 & 0 & 2 & 0 & 0 & 0 & 0 & 2 \\
\hline & 1 & 0 & 1 & 1 & -1 & 1 & 2 & 0 & 3 & 0 & 2 & 0 & 0 & 0 & 0 & 0 & 3 & 0 \\
\hline \multirow[t]{3}{*}{ Figure $9 b$} & 0 & 0 & 1 & -1 & -1 & 0 & 0 & 0 & 2 & 0 & 0 & 0 & 0 & 0 & 0 & 2 & 0 & 0 \\
\hline & 1 & 1 & 1 & 1 & 0 & 2 & 3 & 2 & 2 & 0 & 3 & 0 & 2 & 0 & 0 & 0 & 0 & 2 \\
\hline & 1 & 0 & $1=$ & 1 & -1 & 1 & 3 & 0 & 4 & 1 & 2 & 0 & 0 & 0 & 0 & 0 & 4 & 0 \\
\hline \multirow[t]{3}{*}{ Figure 9c } & 0 & 0 & 1 & -1 & -1 & 0 & 0 & 0 & 2 & 0 & 0 & 0 & 0 & 0 & 0 & 2 & 0 & 0 \\
\hline & 1 & 1 & 1 & 1 & 0 & 2 & 4 & 2 & 3 & 0 & 4 & 0 & 2 & 0 & 0 & 0 & 0 & 3 \\
\hline & 1 & 1 & $1=$ & 1 & 0 & 1 & 1 & 2 & 2 & 0 & 1 & 0 & 2 & 0 & 0 & 0 & 2 & 0 \\
\hline \multirow[t]{3}{*}{ Figure $9 d$} & 0 & 0 & 1 & -1 & -1 & 0 & 0 & 0 & 2 & 0 & 0 & 0 & 0 & 0 & 0 & 2 & 0 & 0 \\
\hline & 1 & 1 & 1 & 2 & 1 & 2 & 2 & 2 & 1 & 0 & 0 & 2 & 0 & 2 & 0 & 0 & 0 & 1 \\
\hline & 1 & 1 & $1=$ & 1 & 1 & 1 & 1 & 2 & 2 & 0 & 1 & 0 & 0 & 2 & 0 & 0 & 2 & 0 \\
\hline \multirow[t]{3}{*}{ Figure 9e } & 0 & 0 & 1 & -1 & -1 & 0 & 0 & 0 & 2 & 0 & 0 & 0 & 0 & 0 & 0 & 2 & 0 & 0 \\
\hline & 1 & 1 & 1 & 2 & 1 & 2 & 2 & 2 & 1 & 0 & 0 & 2 & 0 & 2 & 0 & 0 & 0 & 1 \\
\hline & 1 & 1 & $1=$ & 1 & 1 & 1 & 2 & 3 & 2 & 0 & 2 & 0 & 1 & 2 & 0 & 0 & 2 & 0 \\
\hline \multirow[t]{3}{*}{ Figure $9 f$} & 0 & 0 & 1 & -1 & - & 0 & 0 & 0 & 2 & 0 & 0 & 0 & 0 & 0 & 0 & 2 & 0 & 0 \\
\hline & 1 & 1 & 1 & 2 & 1 & 2 & 3 & 3 & 1 & 0 & 0 & 3 & 0 & 3 & 0 & 0 & 0 & 1 \\
\hline & 1 & 1 & $1=$ & 2 & 1 & 2 & 1 & 1 & 1 & 0 & 0 & 1 & 0 & 1 & 0 & 0 & 0 & 1 \\
\hline \multirow[t]{3}{*}{ Figure $9 g$} & 1 & 1 & 1 & 1 & 0 & 1 & 1 & 2 & 1 & 0 & 1 & 0 & 2 & 0 & 0 & 0 & 1 & 0 \\
\hline & 1 & 1 & 1 & 2 & 1 & 2 & 1 & 1 & 1 & 0 & 0 & 1 & 0 & 1 & 0 & 0 & 0 & 1 \\
\hline & 1 & 1 & $1=$ & 2 & 1 & 2 & 1 & 1 & 1 & 0 & 0 & 1 & 0 & 1 & 0 & 0 & 0 & 1 \\
\hline \multirow[t]{3}{*}{ Figure $9 \mathrm{~h}$} & 1 & 1 & 1 & 1 & 1 & 1 & 1 & 2 & 1 & 0 & 1 & 0 & 0 & 2 & 0 & 0 & 1 & 0 \\
\hline & 1 & 1 & 1 & 2 & 1 & 2 & 1 & 1 & 1 & 0 & 0 & 1 & 0 & 1 & 0 & 0 & 0 & 1 \\
\hline & 1 & 1 & $1=$ & 2 & 1 & 2 & 1 & 1 & 1 & 0 & 0 & 1 & 0 & 1 & 0 & 0 & 0 & 1 \\
\hline \multirow{2}{*}{ Figure $9 \mathrm{i}$} & 1 & 1 & 1 & 1 & 1 & 1 & 2 & 3 & 1 & 0 & 2 & 0 & 1 & 2 & 0 & 0 & 1 & 0 \\
\hline & 1 & 1 & 1 & 2 & 1 & 2 & 2 & 2 & 1 & 0 & 0 & 2 & 0 & 2 & 0 & 0 & 0 & 1 \\
\hline
\end{tabular}

The formalisms of the 9IM for Figure 9a-c, for Figure 9d-f and for Figure 9g-i are the same. Because it considers only the empty and non-empty values, the 9IM concisely represents topological relations. Although there are obvious differences between the topological relations in these figures, using the 9IM, their formalisms are identical.

Because the dimensions of the intersections between the interior of the two lines in Figure $9 a, b$ are zero and one, respectively, the formalisms of the DE-9IM for Figure 9a,b are different. Because the dimensions of the intersections between the interior of the line and the boundary of the region in Figure 9d,e are zero and one, respectively, the formalisms of the DE-9IM for Figure 9d,e are different. Because the dimensions of the intersections between the boundaries of the two regions in Figure $9 \mathrm{~g}, \mathrm{~h}$ are zero and one, respectively, the formalisms of the DE-9IM for Figure 9g,h are different. The DE-9IM is a refinement of the 9IM and can distinguish the dimensions of the intersections. However, the DE-9IM has difficulty distinguishing other topological invariants. For instance, although the topological relations are different in Figure 9b,c, the formalisms of the DE-9IM for Figure 9b,c are the same. Therefore, although the DE-9IM can distinguish the dimensions of intersections; it cannot describe the number of separations.

The number of separations between the interiors of the two lines in Figure $9 b, c$ are two and three, respectively; therefore, the formalisms of the SNE-9IM for those two images are different. The conclusion can be made that the number of separations between the interior of two lines can be any integer greater than or equal to zero, and countless topological relations exist for two lines. The number of separations between the interior of the line and the boundary of the region in Figure $9 \mathrm{e}, \mathrm{f}$ 
are two and three, respectively; therefore, the formalisms of the SNE-9IM for those two images are different. The conclusion can be made that the number of separations between the interior of the line and the boundary of the region can be any integer greater than or equal to zero, and countless topological relations exist for a line and a region. The number of separations between the boundary of two regions in Figure 9h,i are two and three, respectively; therefore, the formalisms of the SNE-9IM for those two images are different. The conclusion can be made that the number of separations between the boundaries of two regions can be any integer greater than or equal to zero, and countless topological relations exist for two regions. The SNE-9IM is a refinement of the 9IM that adds the ability to distinguish the number of separations. However, the SNE-9IM has difficulty distinguishing other topological invariants. For example, although the topological relations are different in Figure $9 a, b$, the formalisms of the SNE-9IM for those two images are the same. Therefore, although the SNE-9IM can distinguish the number of separations, it cannot describe the dimensions of intersections.

Both the DE-9IM and the SNE-9IM can distinguish more topological relations than the 9IM. Compared with the SNE-9IM, the DE-9IM can distinguish some topological relations that cannot be distinguished by the SNE-9IM and vice versa. The 27IM is also a refinement of the 9IM, and it has the characteristics of both the DE-9IM and SNE-9IM. For example, the formalisms of the 27IM for Figure $9 \mathrm{a}-\mathrm{c}$ are different; the formalisms of the 27IM for Figure $9 \mathrm{~d}-\mathrm{f}$ are different; and the formalisms of the 27IM for Figure 9g-i are different. The conclusion can be drawn that the 27IM can distinguish more topological relations than can the 9IM, DE-9IM or SNE-9IM.

\subsection{The Interoperability of the 27IM}

The 27IM is a refinement of the 9IM, DE-9IM and SNE-9IM. The value of the 9IM, DE-9IM and SNE-9IM can be easily derived from the value of the 27IM. Taking the intersection of $A^{\prime}$ s interior and $B^{\prime}$ s interior as an example, $\chi_{0}\left(A^{o} \cap B^{o}\right), \chi_{1}\left(A^{o} \cap B^{o}\right)$ and $\chi_{2}\left(A^{o} \cap B^{o}\right)$ are separately the number of separations in zero dimensions, one dimension and two dimension between $A^{\prime}$ s interior and $B^{\prime} \mathrm{s}$ interior of the 27IM; $\chi\left(A^{o} \cap B^{o}\right)$ is the number of separations between $A^{\prime}$ s interior and $B^{\prime}$ s interior of the SNE-9IM; $\operatorname{dim}\left(A^{o} \cap B^{o}\right)$ is the dimension between $A^{\prime}$ s interior and $B^{\prime}$ s interior of the DE-9IM; and $\operatorname{ct}\left(A^{o} \cap B^{o}\right)$ is the value of the intersection between $A^{\prime}$ s interior and $B^{\prime}$ s interior of the 9IM.

The value of the intersection of $A^{\prime}$ 's interior and $B^{\prime}$ s interior of the SNE-9IM can be derived from the value of the 27IM according to Equation (13). The number of separations of the SNE-9IM is the sum of the number of separations in zero dimensions, one dimension and two dimensions of the 27IM.

$$
\chi\left(A^{o} \cap B^{o}\right)=\chi_{0}\left(A^{o} \cap B^{o}\right)+\chi_{1}\left(A^{o} \cap B^{o}\right)+\chi_{2}\left(A^{o} \cap B^{o}\right)
$$

The value of the intersection of $A^{\prime}$ s interior and $B^{\prime}$ s interior of the DE-9IM can be derived from the value of the 27IM according to Equation (14). The value of the intersection of the DE-9IM is assigned to the maximum of the dimensions of the intersection. If the separation number of the 27IM is greater than zero in two dimensions, the value of the intersection of the DE-9IM is assigned to two. If the separation number of the 27IM is equal to zero in two dimensions and greater than zero in one dimension, the value of the intersection of the DE-9IM is assigned to one. If the separation number of the 27IM is equal to zero in two dimensions and one dimension and greater than zero in zero dimensions, the value of the intersection of the DE-9IM is assigned to zero. If the number of separations is equal to zero in two dimensions, one dimension and zero dimensions, the value of the intersection of the DE-9IM is assigned to -1 .

$$
\operatorname{dim}\left(A^{o} \cap B^{o}\right)= \begin{cases}2 & \operatorname{if}\left(\chi_{2}\left(A^{o} \cap B^{o}\right)>0\right) \\ 1 & \operatorname{if}\left(\chi_{2}\left(A^{o} \cap B^{o}\right)=0 \text { and } \chi_{1}\left(A^{o} \cap B^{o}\right)>0\right) \\ 0 & \operatorname{if}\left(\chi_{2}\left(A^{o} \cap B^{o}\right)=0 \text { and } \chi_{1}\left(A^{o} \cap B^{o}\right)=0 \text { and } \chi_{0}\left(A^{o} \cap B^{o}\right)>0\right) \\ -1 & \operatorname{if}\left(\chi_{2}\left(A^{o} \cap B^{o}\right)=0 \text { and } \chi_{1}\left(A^{o} \cap B^{o}\right)=0 \text { and } \chi_{0}\left(A^{o} \cap B^{o}\right)=0\right)\end{cases}
$$


The value of the intersection of $A^{\prime}$ s interior and $B^{\prime}$ s interior of the 9IM can be derived from the value of the 27IM according to Equation (15). If the number of separations of the 27IM is equal to one in two dimensions, or one dimension or zero dimensions, the value of the intersection of the 9IM is assigned to one. If the number of separations of the 27IM is equal to zero in two dimensions, one dimension and zero dimensions, the value of the intersection of the 9IM is assigned to zero.

$$
c t\left(A^{o} \cap B^{o}\right)= \begin{cases}1 & \operatorname{if}\left(\chi_{2}\left(A^{o} \cap B^{o}\right)=1 \text { or } \chi_{1}\left(A^{o} \cap B^{o}\right)=1 \text { or } \chi_{0}\left(A^{o} \cap B^{o}\right)=1\right) \\ 0 & \operatorname{if}\left(\chi_{2}\left(A^{o} \cap B^{o}\right)=0 \text { and } \chi_{1}\left(A^{o} \cap B^{o}\right)=0 \text { and } \chi_{0}\left(A^{o} \cap B^{o}\right)=0\right)\end{cases}
$$

The interoperability of the 27IM with the SNE-9IM, DE-9IM and 9IM are separately given by Equations (13)-(15). The conclusion can be drawn that the interoperability of the 27IM with other topological relation models, such as the 9IM, DE-9IM and SNE-9IM, is very good.

\section{Conclusions}

The major novel contribution of this paper lies in its proposal of a comprehensive model for topological relations called the 27IM, which considers both the dimension and the number of separations. The 27IM describes six groups of topological relations: point/point, point/line, point/region, line/line, line/region and region/region relations. The first advantage of the 27IM is that the topological relations are preserved under topological transformations. The second advantage of the 27IM is that it distinguishes further topological relations compared with the 9IM, DE-9IM and SNE-9IM. The third advantage of the 27IM is that the interoperability of the 27IM with the 9IM, DE-9IM and SNE-9IM is good.

Although the 27IM is a detailed model, it is more complex than the 9IM, DE-9IM and SNE-9IM, which each requiring only a nine-tuple, while the 27IM requiring a 27-tuple. It is inevitable that many invalid spatial topological relations appear in the 27IM. To exclude impossible spatial topological relations, the 23 conditions proposed by Egenhofer and Herring (1991) can be revised for the 27IM. It is impossible to contain all of the details for the 27IM. Some other topological invariants (i.e., the sequence of boundary-boundary intersection or the types of boundary-boundary intersections) are not considered. The 27IM can be extended to represent more topological relation details whenever necessary. This study addresses only simple objects; complex objects, such as multi-point, multi-line and multi-region objects, are not considered. As is common knowledge, complex objects can be considered as combinations of simple objects. Therefore, the 27IM can also describe the topological relations between complex objects. The numbers of possible topological relations involved in line/line, line/region and region/region relations are countless; consequently, only some typical relations and their corresponding geometric interpretations are included. Only two-dimensional spatial objects are discussed; three-dimensional spatial objects are not included. Although the 27IM cannot be directly used for three-dimensional spatial objects, the 27IM can be extended to the 36-intersection model (36IM) for three-dimensional spatial object. The $36 \mathrm{IM}$ would be a $12 \times 3$ matrix, which represents the number of the separations in zero dimensions, one dimension, two dimensions and three dimensions.

Finally, the 27IM could distinguish further topological relations based on the recognition of the dimension and the number of separations. Other topological invariants as refinements of the empty/non-empty specifications will be included in the future. The topological relations of three-dimensional objects and complex objects will also be discussed.

Acknowledgments: This research was jointly supported by the National Natural Science Foundation of China (No. 41301417), the Chongqing Natural Science Foundation (No. cstc2014jcyjA20017) and the Fundamental Research Funds for the Central Universities (No. XDJK2013C035). We would like to thank the editors and anonymous referees for their constructive comments.

Author Contributions: The experiments were carried out by Jingwei Shen, who also prepared the figures. This manuscript was written by Jingwei Shen, Tinggang Zhou and Min Chen.

Conflicts of Interest: The authors declare no conflict of interest. 


\section{References}

1. Chen, J.; Li, C.; Li, Z.; Gold, C. A Voronoi-based 9-intersection model for spatial relations. Int. J. Geogr. Inf. Sci. 2001, 3, 201-220. [CrossRef]

2. Landau, B.; Jackendoff, R. "What" and "Where" in spatial language and spatial cognition. Behav. Brain. Sci. 1993, 2, 217-238. [CrossRef]

3. Egenhofer, M.; Mark, D. Naive geography. In Proceedings of the COSIT '95, Semmering, Austria, 21-23 September 1995; pp. 1-15.

4. Dube, M.; Egenhofer, M. An ordering of convex topological relations. In Proceedings of the GIScience, Columbus, OH, USA, 18-21 September 2012; pp. 72-86.

5. Allen, J. Maintaining knowledge about temporal intervals. Commun. ACM. 1983, 11, 832-843. [CrossRef]

6. Masunaga, Y. Re-examination of Allen's interval-based temporal logic. Trans. Inform. Process. Soc. Jpn. 1998, 4, 846-855.

7. Egenhofer, M.; Franzosa, R. Point-set topological spatial relations. Int. J. Geogr. Inf. Sci. 1991, 2, 161-174. [CrossRef]

8. Egenhofer, M.; Herring, J. Categorizing Binary Topological Relations between Regions, Lines and Points in Geographic Databases; Technical Report 01/1991; University of Maine: Orono, ME, USA, 1991; p. 28.

9. Egenhofer, M.; Sharma, J.; Mark, D. A critical comparison of the 4-intersection and 9-intersection models for spatial relations: Formal analysis. In Proceedings of the AutoCarto Conference, Minneapolis, MN, USA, 30 October-1 November 1993; pp. 1-12.

10. Randell, D.; Cohn, A. Modelling topological and metrical properties of physical processes. In Proceedings of the International Conference on Principles of Knowledge Representation and Reasoning, Toronto, ON, Canada, 15-18 May 1989; pp. 357-368.

11. Randell, D.; Cui, Z.; Cohn, A. A spatial logic based on regions and connection. In Proceedings of the 3rd International Conference on Principles of Knowledge Representation and Reasoning; Morgan Kaufmann Publishers Inc.: San Francisco, CA, USA, 1992; pp. 165-176.

12. Cohn, A.; Randell, D.; Cui, Z. Taxonomies of logically defined qualitative spatial relations. Int. J. Hum. Comput. St. 1995, 5, 831-846. [CrossRef]

13. Gotts, N.; Gooday, J.; Cohn, A. A connection based approach to common-sense topological description and reasoning. Monist 1996, 1, 51-75. [CrossRef]

14. Cohn, A.; Bennett, B.; Gooday, J.; Gotts, N. RCC: A calculus for region based qualitative spatial reasoning. GeoInformatica 1997, 3, 275-316. [CrossRef]

15. Jonsson, P.; Drakengren, T. A complete classification of tractability in RCC-5. J. Artif. Intell. Res. 1997, 1, 211-221.

16. Deng, M.; Cheng, T.; Chen, X.; Li, Z. Multi-level topological relations between spatial regions based upon topological invariants. Geoinformatica 2007, 11, 239-267. [CrossRef]

17. Liu, K.; Shi, W. Extended model of topological relations between spatial objects in geographic information systems. Int. J. Appl. Earth. Obs. 2007, 9, 264-275. [CrossRef]

18. Kurata, Y. The 9+-intersection: A universal framework for modeling topological relations. In Geographic Information Science; Springer: Berlin/Heidelberg, Germany, 2008; pp. 181-198. [CrossRef]

19. Alboody, A.; Sedes, F.; Inglada, J. Modeling topological relations between uncertain spatial regions in geo-spatial databases: Uncertain intersection and difference topological model. In Proceedings of the 2010 Second International Conference on Advances in Databases, Knowledge, and Data Applications, Menuires, France, 11-16 April 2010; pp. 7-15.

20. Leng, L.; Yang, G.; Chen, S. A combinatorial reasoning mechanism with topological and metric relations for change detection in river planforms: An application to globeland30's water bodies. ISPRS Int. J. Geo-Inf. 2017, 1, 1-13. [CrossRef]

21. Long, Z.; Li, S. A complete classification of spatial relations using the voronoi-based nine-intersection model. Int. J. Geogr. Inf. Sci. 2013, 10, 2006-2025. [CrossRef]

22. Lewis, J.; Dube, M.; Egenhofer, M. The topology of spatial scenes in R2. In Proceedings of the COSIT 2013, Scarborough, UK, 2-6 September 2013; pp. 495-515. 
23. Dube, M.; Egenhofer, M.; Lewis, J.; Stephen, S.; Plummer, M. Swiss canton regions: A model for complex objects in geographic partitions. In Proceedings of the COSIT 2015, Santa Fe, NM, USA, 12-16 October 2015; pp. 309-330.

24. Egenhofer, M. A Reference System for Topological Relations between Compound Spatial Objects. In Advances in Conceptual Modeling —Challenging Perspectives; Heuser, C., Pernul, G., Eds.; Springer: Berlin, Germany, 2009; Volume 5833, pp. 307-316.

25. Clementini, E.; Felice, P.; Oosterom, P. A small set of formal topological relationships suitable for end-user interaction. In Proceedings of the International Symposium Ssd'93 Lecture Notes in Computer Science, Singapore, 23-25 June 1993; pp. 277-295.

26. Egenhofer, M. A model for detailed binary topological relationships. Geomatica 1993, 3, 261-273.

27. Egenhofer, M.; Franzosa, R. On the equivalence of topological relations. Int. J. Geogr. Inf. Sci. 1995, 2, $133-152$. [CrossRef]

28. Clementini, E.; Felice, P. Topological invariants for lines. IEEE. Trans. Knowl. Data. Eng. 1998, 1, 38-54. [CrossRef]

29. Egenhofer, M.; Shariff, A. Metric details for natural-language spatial relations. ACM Trans. Inf. Syst. 1998, 4, 321-349. [CrossRef]

30. Shariff, A.; Egenhofer, M.; Mark, D. Natural-language spatial relations between linear and areal objects: the topology and metric of English-language terms. Int. J. Geogr. Inf. Sci. 1998, 3, 215-246.

31. Godoy, F.; Rodríguez, A. A quantitative description of spatial configurations. In Advances in Spatial Data Handling; Springer: Heidelberg, Germany, 2002; pp. 299-311.

32. Nedas, K.; Egenhofer, M.; Wilmsen, D. Metric details of topological line-line relations. Int. J. Geogr. Inf. Sci. 2007, 1, 21-48. [CrossRef]

33. Egenhofer, M.; Dube, M. Topological relations from metric refinements. In Proceedings of the 17th ACM Sigspatial International Symposium on Advances in Geographic Information Systems, Seattle, WA, USA, 4-6 November 2009; pp. 158-167.

34. Sridhar, M.; Cohn, A.; Hogg, D. From video to RCC8: Exploiting a distance based semantics to stabilise the interpretation of mereotopological relations. In Spatial Information Theory; Springer: Heidelberg, Germany, 2011; pp. 110-125.

35. Dube, M.; Barrett, J.; Egenhofer, M. From metric to topology: Determining relations in discrete space. In Proceedings of the 12th International Conference, COSIT 2015, Santa Fe, NM, USA, 12-16 October 2015; pp. 151-171.

36. Carlson, E. Three dimensional conceptual modeling of subsurface structures. In Proceedings of the ASPRS-ACSM Annual Convention, Baltimore, MD, USA, 29 March-3 April 1987; pp. 188-200.

37. Molenaar, M. A formal data structure for 3D vector maps. In Proceedings of the EGIS'90, Amsterdam, The Netherlands, 10-14 September 1990; pp. 770-781.

38. Pilouk, M.; Tempfli, K.; Molenaar, M. A tetrahedron-based 3D vector data model for geo-information. In Proceedings of the AGDM'94 Spatial Data Modelling and Query Languages for 2D and 3D Applications, Delft, The Netherlands, 12-14 September 1994; pp. 129-140.

39. Pilouk, M. Integrated Modelling for 3D GIS. Ph.D. Thesis, The International Institute for Aerospace Survey and Earth Sciences (ITC), Enschede, The Netherlands, June 1996.

40. Pigot, S. Topological models for 3D spatial information systems. In Proceedings of the AutoCarto Conference, Baltimore, MD, USA, 25-28 March 1991; pp. 368-392.

41. Brisson, E. Representing geometric structures ind dimensions: Topology and order. Discrete. Comput. Geom. 1993, 1, 387-426. [CrossRef]

42. Open GIS Consortium, Inc. OpenGIS simple features specification for SQL (Revision 1.1). 1999. Available online: http:/ /www.opengis.org/techno/specs/99--049.pdf (accessed on 15 May 2016).

43. Zlatanova, S. 3D GIS for Urban Development. Ph.D. Thesis, The International Institute for Aerospace Survey and Earth Sciences (ITC), Enschede, The Netherlands, March 2000.

44. Ramos, F. A multi-level approach for 3D modeling geographical information sytems. In Proceedings of the Symposium on Geospatial Theory, Processing and Applications, Ottawa, ON, Canada, 9-12 July 2002; pp. $1-5$.

45. Lee, J.; Kwan, M. A combinatorial data model for representing topological relations among 3D geographical features in micro-spatial environments. Int. J. Geogr. Inf. Sci. 2005, 10, 1039-1056. [CrossRef] 
46. Open Geospatial Consortium. OGC City Geography Markup Language (CityGML) Encoding Standard. 2012. Available online: https:/ / portal.opengeospatial.org/ files/ ?artifact_id=47842 (accessed on 7 July 2016).

47. Yuan, L.; Yu, Z.; Luo, W.; Yi, L.; Lv, G. Multidimensional-unified topological relations computation: A hierarchical geometric algebra-based approach. Int. J. Geogr. Inf. Sci. 2014, 12, 2435-2455. [CrossRef]

48. Yu, Z.; Luo, W.; Yuan, L.; Hu, Y.; Zhu, A.; Lv, G. Geometric algebra model for geometry-oriented topological relation computation. Trans. GIS 2016, 2, 259-279. [CrossRef]

49. Adams, C.; Franzosa, R. Introduction to Topology: Pure and Applied; Prentice Hall: Upper Saddle River, NJ, USA, 2008; pp. 73-83.

(C) 2017 by the authors; licensee MDPI, Basel, Switzerland. This article is an open access article distributed under the terms and conditions of the Creative Commons Attribution (CC BY) license (http:/ / creativecommons.org/licenses/by/4.0/). 